\title{
Limitações e controvérsias na determinação do valor preditivo de critérios de morfologia oocitária e embrionária
}

\author{
Limitations and controversies in determining the predictive \\ value of oocyte and embryo morphology criteria
}

Artigo de Revisão

\section{Palavras-chave \\ Infertilidade \\ Fertilização in vitro \\ Desenvolvimento embrionário Transferência embrionária \\ Keywords \\ Infertility \\ In vitro fertilization \\ Embryo development \\ Embryo transfer}

\section{Resumo}

Com o objetivo de aumentar as taxas de sucesso das pacientes que são submetidas a técnicas de reprodução humana assistida (RHA), numerosos estudos apresentam como foco a identificação do embrião com maior potencial de implantação. Apesar dos avanços tecnológicos significativos da Medicina Reprodutiva baseados no advento da era da genômica, proteômica e metabolômica (OMICS), técnicas rotineiramente aplicáveis ainda não estão disponíveis. Dessa forma, laboratórios de fertilização in vitro (FIV) de todo o mundo selecionam para transferência embriões humanos cultivados in vitro baseados em parâmetros morfológicos avaliados em microscopia de luz. Diversos parâmetros morfológicos podem ser avaliados desde o estágio pronuclear até o estágio de blastocisto para embriões humanos cultivados in vitro. De modo geral, independentemente do dia da transferência, tais critérios parecem apresentar valor preditivo de viabilidade embrionária quando avaliados individualmente ou coletivamente. No entanto, a subjetividade da avaliação morfológica, bem como a ampla diversidade de sistemas de classificação embrionária aplicados por diferentes clínicas, implica em resultados contraditórios, tornando extremamente difícil a implementação de um consenso do valor preditivo dos diferentes parâmetros morfológicos avaliados. A otimização da seleção embrionária representa um grande potencial de aumento das taxas de sucesso do tratamento, além de possibilitar a realização da transferência de um número reduzido de embriões, minimizando os riscos derivados de gestações múltiplas.

\section{Abstract}

In order to increase the success rate of in vitro fertilization cycles, several studies have focused on the identification of the embryo with higher implantation potential. Despite recent advances in the reproductive medicine, based on the OMICs technology, routinely applicable methodologies are still needed. Thus, in most fertilization centers embryo selection for transfer is still based on morphological parameters evaluated under light microscopy. Several morphological parameters may be evaluated, ranging from the pronuclear to blastocyst stage. In general, despite the day of transfer, some criteria are suggested to present a predictive value for embryo viability when analyzed independently or combined. However, the subjectivity of morphological evaluation, as well as the wide diversity of embryo classification systems used by different fertilization centers shows contrasting results, making the implementation of a consensus regarding different morphological criteria and their predictive value a difficult task. The optimization of embryo selection represents a large potential to increase treatment success rates, allowing the transfer of a reduced number of embryos and minimizing the risks of multiple pregnancy.

\section{Correspondêncii \\ Rita de Cássia Savio Figueira Av. Brigadeiro Luís Antônio, 4.545 - Jardim Paulisto CEP: 01401-002 \\ São Paulo (SP), Brasil \\ Recebido \\ $11 / 03 / 2015$ \\ Aceito com modificacọoes \\ $17 / 07 / 2015$}

Centro de Fertilização Assistida Fertility - São Paulo (SP), Brasil.

'Faculdade de Ciências Médicas da Santa Casa de Misericórdia de São Paulo - FCMSCSP - São Paulo (SP), Brasil; Centro de Fertilização Assistida Fertility, Centro de Ensino e Pesquisa em Reprodução Assistida, Instituto Sapientiae - São Paulo (SP), Brasil. 2Departamento de Ginecologia e Obstetrícia, Faculdade de Ciências Médicas da Santa Casa de São Paulo - FCMSCSP - São Paulo (SP), Brasil.

${ }^{3}$ Centro de Fertilização Assistida Fertility, Centro de Ensino e Pesquisa em Reprodução Assistida, Instituto Sapientiae - São Paulo (SP), Brasil. Conflito de interesses: não há. 
Introdução

\section{Reprodução humana assistida}

Evidências epidemiológicas sugerem que a infertilidade acomete por volta de $15 \%$ dos casais em idade reprodutiva. Segundo o mais recente registro do Centro de Controle e Prevenção de Doenças (CDC) dos Estados Unidos da América (EUA), a partir de dados compilados pela Pesquisa Nacional de Crescimento Familiar (NSFG), em 2010, dentre aproximadamente 62 milhões de mulheres com idade entre 15 e 44 anos, cerca de 7,4 milhões, ou $12 \%$, receberam tratamento para infertilidade em algum momento de suas vidas. Dentre as mulheres casadas de mesma faixa etária estima-se que 1,5 milhão sejam inférteis ${ }^{1}$. Em registro publicado em 2014, referente às taxas de sucesso obtidas por centros de fertilização assistida em 2012, o CDC divulgou a realização de 176.247 procedimentos de reprodução humana assistida (RHA) por 456 clínicas, que seriam representativas de $98 \%$ dos tratamentos realizados no país ${ }^{2}$. Segundo o último registro da Sociedade Europeia de Reprodução Humana e Embriologia (ESHRE), 550.296 procedimentos de RHA foram relatados por 991 clínicas de 31 países em 2010. Estima-se que tenham sido realizados 1.221 ciclos por milhão de habitantes ${ }^{3}$. Considerando-se o contexto no qual o Brasil encontra-se inserido, segundo o último Registro Latino-americano (RLA), relato da Rede Latino-americana de Reprodução Assistida (REDLARA), foram reportados 47.326 procedimentos de RHA realizados em 2012 por 155 centros de 14 países. Os dados relatados representam um aumento de $12,9 \%$ no número de ciclos realizados, quando comparado ao ano de $2011^{4}$.

Dentre as técnicas de RHA utilizadas para o tratamento de infertilidade, destacam-se a fertilização in vitro (FIV) clássica e a injeção intracitoplasmática de espermatozoides (ICSI). A ICSI foi introduzida em $1992^{5}$, sendo utilizada exclusivamente em casos de fator masculino de infertilidade, incluindo alterações de concentração, motilidade e morfologia espermática. De fato, por meio da utilização dessa técnica é possível obter taxas satisfatórias de fertilização e gestação em casos que envolvem recuperação cirúrgica de espermatozoides ${ }^{6}$. Relatos recentes indicam que, atualmente, a técnica representa $72,6 \%$ dos procedimentos realizados nos EUA, sendo de $36,4 \%$ o número obtido para o ano de $1996^{2}$. O aumento do uso da técnica está associado a sua aplicação para o tratamento de casais inférteis na ausência de fator masculino ${ }^{7}$. Segundo registro da RLA, a ICSI representou $85 \%$ dos procedimentos de RHA realizados na América Latina, proporção que permanece sem alteração desde $2008^{4}$.

No início da aplicação das técnicas de RHA, baixas taxas de sucesso foram obtidas por embrião transferido.
Naquele momento, o aumento do número de embriões transferidos foi uma estratégia aplicada com o objetivo de se obter um aumento significativo no valor de tais taxas. Ao longo dos anos, maiores taxas de implantação embrionária foram obtidas, porém acompanhadas de taxas elevadas de gestação múltipla relatadas até os dias de hoje ${ }^{2-4}$. Com o objetivo de reduzir os índices de multiparidade, algumas políticas de redução do número de embriões a serem transferidos foram sugeridas ${ }^{8}$. Dessa forma, a seleção do embrião com maior probabilidade de implantação tornou-se um dos principais entraves da RHA e foco de numerosos estudos, que serão abordados posteriormente de acordo com o dia de desenvolvimento embrionário. O presente estudo teve como objetivo descrever os critérios de morfologia oocitária e embrionária até o dia 3 do desenvolvimento previamente abordados na literatura (Quadro 1), de forma a abordar suas possíveis implicações para o desenvolvimento embrionário até o estágio de blastocisto e seu possível potencial preditivo no potencial de implantação destes.

\section{Morfologia oocitária}

$\mathrm{Na}$ rotina da maioria dos laboratórios de FIV, a avaliação morfológica oocitária inicia-se com classificação do grau de expansão das células do complexo cumulus-corona em estereomicroscópio. Considerando-se a remoção dessas células em oócitos que serão submetidos à ICSI, a avaliação mais acurada em microscópio invertido fornece informações a respeito do grau de maturação nuclear, sendo o

Quadro 1. Critérios de morfologia oocitária e embrionária abordados de forma a revisar suas respectivas implicações no desenvolvimento embrionário

\begin{tabular}{|l|}
\hline \multicolumn{1}{|c|}{ Morfologia oocitúría } \\
\hline Dismorfismos intracitoplasmáticos \\
Granulação \\
Inclusões \\
Dismorfismos extracitoplasmáticos \\
Zona pelúcida \\
Espaço perivitelineo \\
Corpúsculo polar \\
Forma \\
\hline Morfologia pronuclear \\
Pronúcleo \\
Corpos precursores de nucléolos \\
Halo citoplasmático \\
Classificacãoo embrionária nos dias 2 e 3 do desenvolvimento \\
Número de células e ritmo de clivagem \\
Simetria de blastômeros \\
Fragmentos anucleados \\
Multinucleação e ausência de núcleos
\end{tabular}


$1^{\circ}$ corpúsculo polar (CP) marcador de maturidade em oócitos em Metáfase II da Meiose II (MII) ${ }^{9}$. Posteriormente, todos os oócitos MII são submetidos à ICSI, sendo o potencial de desenvolvimento embrionário estimado exclusivamente com base na morfologia embrionária, independentemente da qualidade do oócito a partir do qual este foi derivado. No entanto, a qualidade oocitária pode ser considerada um fator limitante da fertilidade feminina, refletindo o potencial de desenvolvimento intrínseco de um oócito, além de apresentar um papel crucial não apenas na fertilização, mas também no desenvolvimento subsequente ${ }^{10}$.

Uma complexa cascata de eventos de maturação que ocorrem durante o desenvolvimento folicular confere ao oócito a capacidade de fertilização e o subsequente desenvolvimento embrionário ${ }^{11}$. A maturação oocitária envolve não apenas alterações relacionadas a eventos nucleares, mas também alterações citoplasmáticas que ocorrem de maneira coordenada, sendo que a maturidade nuclear não pode ser considerada sinônimo de competência oocitária, por não refletir a maturidade citoplasmática ${ }^{12}$. A maturação citoplasmática envolve inúmeros processos moleculares e estruturais responsáveis pela preparação do oócito para os eventos ligados à fertilização. As mudanças estruturais do citoplasma são caracterizadas por uma extensa reorganização de organelas intracelulares, com destaque para a reorganização de mitocôndrias visando o fornecimento de um suprimento local de energia, a migração de grânulos corticais para a periferia do oolema, para bloqueio da polispermia, e a movimentação do retículo endoplasmático (RE), antecipando a liberação de $\mathrm{Ca}^{2+}$ que ocorre durante a fertilização. Dessa forma, deficiências no processo de maturação citoplasmática podem comprometer todos os processos que preparam o oócito para ativação, adequada fertilização e desenvolvimento embrionário. Além das deficiências do processo, a assincronia nos eventos de maturação nuclear e citoplasmática foi associada à ocorrência de dismorfismos oocitários ${ }^{13}$. Oócitos considerados morfologicamente normais devem apresentar: forma esférica; citoplasma transparente moderadamente granular e livre de inclusões; pequeno espaço perivitelineo (EPV), livre de granulações, contendo um único $\mathrm{CP}$ redondo e intacto; zona pelúcida ( $\mathrm{ZP})$ clara e de espessura apropriada $^{14}$. Usualmente, os dismorfismos oocitários podem ser divididos em intra (IC) e extracitoplasmáticos (EC). Os dismorfismos IC envolvem a presença de granulações e/ou inclusões citoplasmáticas, como corpos refráteis, vacúolos (VAC) e/ou agregados do retículo endoplasmático liso (REL). Os dismorfismos EC envolvem anormalidades: na forma do oócito, no EPV, como a presença de grânulos corticais, na ZP, incluindo espessura e coloração, e/ou no $\mathrm{CP}$, como alteração do seu tamanho ${ }^{9,12}$.

A qualidade oocitária pode ser considerada um fator-chave na fertilidade feminina, sendo previamente reportado que o desenvolvimento de anomalias intra e extracitoplasmáticas durante o processo de maturação pode resultar em falha de fertilização ${ }^{15}$, aneuploidia cromossômica $^{16}$ e alteração do desenvolvimento embrionário $^{12}$. Apesar de identificadas as alterações que podem comprometer a competência oocitária, pouco se sabe em relação às suas origens, a quando e como essas alterações podem afetar processos do desenvolvimento normal e ao fato da possibilidade de seus efeitos envolverem a alteração da expressão de vias de sinalização regulatórias ${ }^{17}$.

As variações morfológicas oocitárias podem ser resultado de fatores intrínsecos, como a idade da paciente, e/ou de fatores extrínsecos associados, por exemplo, aos protocolos de estimulação ovariana controlada (EOC) e à resposta da paciente ao tratamento ${ }^{10}$. A maioria dos oócitos recuperados após EOC apresenta uma ou mais variações dos critérios morfológicos ${ }^{12,15}$, sendo o mesmo observado para pacientes férteis doadoras de oócitos ${ }^{18}$.

Relatos do consenso de Istambul de avaliação embrionária ${ }^{17}$ sugerem que dismorfismos EC são variações fenotípicas na maioria das vezes relacionadas às condições de cultivo ou à idade da paciente. Por outro lado, a presença de agregados de REL foi associada ao comprometimento da competência oocitária e relacionada a perdas fetais e distúrbios de imprinting ${ }^{19}$. De modo geral, a presença dos dismorfismos IC e EC e sua associação com a competência oocitária e com o comprometimento do desenvolvimento embrionário é controversa, conforme apontado em estudos recentes de revisão sistemática e metanálise ${ }^{20,21}$.

\section{Dismorfismos intracitoplasmáticos}

\section{Granulação}

O aumento da granulação do citoplasma pode ocorrer de forma homogênea, afetando todo o citoplasma do gameta, ou de localização centralizada, sendo esta última associada a um pior prognóstico ${ }^{22}$. Além da granulação, alterações na viscosidade e na fluidez do citoplasma também foram descritas $^{23}$. A fluidez do citoplasma pode ser avaliada de acordo com a persistência do cone de injeção formado no citoplasma do oócito decorrente entrada da micropipeta de ICSI no momento da injeção, reflexo da deficiência na textura citoplasmática.

\section{Inclusões}

Numerosos tipos de inclusões citoplasmáticas podem ser observados no citoplasma de oócitos avaliados em microscopia de luz. Os corpos refráteis, assim chamados devido a sua aparência quando identificados em microscopia de luz, são estruturas de aproximadamente $10 \mu \mathrm{m}$ de diâmetro compostas de material lipídico e grânulos 
densos. VAC são inclusões citoplasmáticas rodeadas por membrana, podendo variar em número e tamanho. Acreditase que os VAC possam surgir espontaneamente ou por fusão de vesículas derivadas do REL e/ou do complexo de Golgi ${ }^{24}$. Por outro lado, os mecanismos associados com o surgimento dos agregados de REL são desconhecidos. A distinção entre essas estruturas é dada pelo fato de que os agregados de REL são estruturas translúcidas não delimitadas por membrana ${ }^{9,12}$.

Estudos sugerem que a presença de algumas inclusões citoplasmáticas, como os corpos refráteis, não exerce impacto na qualidade do oócito. Por outro lado, a presença de VAC e agregados do REL foi associada ao comprometimento da competência de oócitos humanos ${ }^{12,23}$.

Ainda não foi elucidado de que modo as inclusões citoplasmáticas ou deficiências na fluidez do citoplasma afetariam a viabilidade oocitária. Estudos sugerem que os efeitos estariam relacionados à função do citoesqueleto e à estrutura do fuso meiótico ${ }^{23}$. Dessa forma, defeitos no citoplasma oocitário afetariam a formação e o posicionamento dos pronúcleos, dado que o citoesqueleto poderia não se organizar perfeitamente, comprometendo o posicionamento correto do fuso meiótico durante a retomada da meiose. Além disso, a cascata de eventos da fertilização envolve liberação de cálcio e grânulos corticais, síntese de proteína e alterações do citoesqueleto ${ }^{25}$. Assim, considerando a importância dos fatores presentes no ooplasma nesse processo, fica clara a possibilidade de comprometimento da fertilização por anormalidades oocitárias citoplasmáticas. Por outro lado, relatos sugerem que as perdas fetais e as desordens de imprinting associadas à presença dos agregados de REL estejam associadas aos altos níveis de cálcio observados durante a ativação oocitária, além do fato de os níveis serem mantidos elevados por maior período de tempo ${ }^{26}$.

\section{Dismorfismos extracitoplasmáticos}

\section{Zona pelúcida}

A ZP é uma matriz multilaminar composta por glicoproteínas, polissacarídeos e proteínas específicas, com aproximadamente 15-20 $\mu \mathrm{m}$ de espessura, que envolve o oócito e o embrião no início do desenvolvimento ${ }^{27}$. A estrutura da ZP é formada durante a maturação folicular pelo oócito e, em parte, pelas células da granulosa. A formação e a organização das proteínas específicas da ZP durante a maturação oocitária podem refletir a competência oocitária ${ }^{28}$. Segundo consenso, não há benefício na determinação da espessura da ZP em microscopia de luz. Porém, considerando-se a possibilidade de efeitos paciente-específico sugere-se o registro de alterações na espessura ou coloração dessa estrutura ${ }^{17}$.

\section{Espaço perivitelineo}

O EPV é uma estrutura fluida que se encontra entre a membrana plasmática do oócito, chamada oolema, e a ZP. O EPV pode variar em tamanho e conteúdo, sendo o tamanho do EPV associado à fase de maturação oocitária. Nos oócitos maduros o EPV apresenta seu maior grau de expansão, quando comparado aos oócitos imaturos ${ }^{29}$.

Duas hipóteses foram propostas para explicar a presença de grânulos no EPV. A primeira hipótese é derivada de dados de microscopia eletrônica indicando a presença de uma matriz extracelular formada por grânulos e filamentos no espaço entre o oolema e a $\mathrm{ZP}^{30}$. A segunda baseia-se na existência de processos das células da corona que atravessam a ZP do oócito em Prófase I da Meiose I (PI). Acredita-se que, após a remoção desses processos, alguns resquícios permaneçam no EPV ${ }^{31}$. A presença de grânulos parece estar também associada à dose de gonadotrofina administrada durante o EOC $^{32}$.

\section{Corpúsculo polar}

$\mathrm{O} 1{ }^{\circ} \mathrm{CP}$ é uma célula-filha, contendo 23 cromossomos e 46 cromátides, gerada após a telófase da primeira divisão meiótica. Alguns critérios morfológicos do $1^{\circ} \mathrm{CP}$, como a forma, o tamanho e a integridade, podem ser utilizados na predição da qualidade do oócito ${ }^{12}$. Considerando-se a dependência do fator tempo, postula-se que a morfologia do $1^{\circ} \mathrm{CP}$ possa fornecer informações a respeito da idade pós-ovulatória do oócito. Apesar de alguns CPs permanecerem intactos por mais de 20 horas após a ovulação, em geral após esse intervalo tais estruturas apresentam sinais de fragmentação e degeneração. $\mathrm{O}$ aumento de tamanho do $1^{\circ} \mathrm{CP}$ pode ser considerado o mais importante dismorfismo associado ao $\mathrm{CP}$, estando relacionado ao aumento da incidência de aneuploidiais nos oócitos ${ }^{33}$.

\section{Forma}

Alterações na forma dos oócitos são acompanhadas de irregularidades na estrutura e na composição da ZP. Dois possíveis mecanismos foram sugeridos para a ocorrência de oócitos com alteração de forma: o estresse mecânico durante o processo da punção folicular e/ou denudação, deformando tanto o citoplasma quanto a ZP do oócito (com possível recuperação de forma em 24 horas), e uma anomalia preexistente gerada durante a maturação intrafolicular ${ }^{34}$. A diminuição dos pontos de contato célula-célula em embriões alongados, requisito essencial para o desenvolvimento embrionário e a formação da blastocele, justificaria o impacto negativo desse dismorfismo na competência oocitária ${ }^{35}$.

Assim, uma análise da literatura demonstra que o efeito de alterações da morfologia oocitária nas taxas de sucesso após ICSI permanece controverso ${ }^{20,21}$, fazendo com que exista uma tendência de não se considerar a 
morfologia oocitária no processo de decisão durante a seleção de embriões ou blastocistos para transferência. Considerando-se que muitos dismorfismos oocitários são avaliados em conjunto, e não individualmente, ou não são avaliados em muitos desses estudos, o papel negativo das alterações de morfologia oocitária na competência embrionária ainda não foi elucidado. Além disso, o impacto da qualidade oocitária no desenvolvimento embrionário pré-implantacional parece estar relacionado ao número e ao grau de extensão dos dismorfismos, dificultando a realização de estudos comparativos ${ }^{17}$. Em contraste com os processos in vivo, nos quais a maturação ocorre como resultado de um longo e meticuloso processo de seleção natural $^{36}$, procedimentos de estimulação ovariana suprimem esse mecanismo de seleção e permitem a maturação de oócitos de qualidade comprometida de forma inerente, destinados à falha de fertilização e ao desenvolvimento embrionário comprometido ${ }^{14}$. A qualidade oocitária é determinada não apenas pelo genoma nuclear e mitocondrial, mas também pelo microambiente do ovário e do folículo pré-ovulatório capaz de modificar a transcrição gênica e a tradução de proteínas. Avaliando esse complexo cenário, torna-se difícil acreditar que um simples fator, característica ou mecanismo possa de forma adequada indicar a competência oocitária. Uma análise complexa baseada em sistemas de pontuação parece ser uma estratégia promissora, porém resultados contraditórios também foram observados nesses estudos, dadas as variações nos sistemas de pontuação ${ }^{15,37}$.

\section{Morfologia embrionária}

A seleção de embriões de maior potencial para implantação pode ser considerada um dos mais importantes entraves da RHA. Apesar dos avanços tecnológicos significativos da Medicina Reprodutiva baseados no advento da era do OMICS (conjunto de ferramentas de análise genômica, transcriptômica e proteômica) e do estudo da cinética embrionária por meio da tecnologia de time-lapse, a maioria dos laboratórios de FIV de todo o mundo seleciona para transferência embriões humanos cultivados in vitro baseada em parâmetros morfológicos avaliados pontualmente em microscopia de luz.

Recentemente, sistemas de imagem time-lapse foram rapidamente integrados a laboratórios de FIV nos últimos cinco $\operatorname{anos}^{38}$. Os sistemas de monitoramento time-lapse registram imagens digitais dos embriões em cultivo em intervalos de tempo predeterminados. Os sistemas podem ser instalados em incubadoras convencionais de cultivo embrionário ou podem ser adquiridos sistemas combinados de incubadoras time-lapse. As imagens obtidas são compiladas por sistemas computacionais especializados que fornecem uma sequência em time-lapse de todo o desenvolvimento embrionário, dessa forma excluindo a necessidade de retirada do embrião da incubadora pelo embriologista para avaliação de sua morfologia. A aquisição dessa tecnologia envolve investimento significativo, sendo três as principais tecnologias disponibilizadas no mercado: Embryoscope $^{\circledR}$ (Fertilitech), Primo Vision (Vitrolife) e Eeva (Auxogyn, Inc.) ${ }^{39}$. As vantagens propostas, como, por exemplo, a possibilidade de cultivo ininterrupto, a otimização da documentação dos procedimentos, o controle de qualidade e, especialmente, a introdução de novos marcadores de qualidade embrionária, têm estimulado o interesse pela tecnologia ${ }^{40}$. Um grande número de publicações tem sugerido que o intervalo de tempo no desenvolvimento difere entre embriões viáveis e não viáveis ${ }^{38}$, porém poucos relatos disponibilizam modelos aplicáveis de seleção embrionária ${ }^{41}$. Além disso, estudos multicêntricos têm demonstrado as limitações desses modelos quando aplicados por diferentes clínicas ${ }^{42}$. Dessa forma, evidências a respeito do aumento significativo das taxas de nascidos vivos e da efetividade de custo da implementação dessa tecnologia em laboratórios de FIV têm sido questionadas ${ }^{43}$.

A metabolômica e a proteômica são, dentre as tecnologias OMICS, as plataformas mais promissoras, dado o seu caráter não invasivo de avaliação da qualidade embrionária. Na metabolômica é possível mensurar o consumo e a produção de múltiplos substratos pelo embrião analisando o meio de cultivo ao qual o embrião foi exposto durante o procedimento in vitro ${ }^{44}$. O proteoma representa todas as proteínas responsáveis pelas funções celulares, traduzidas a partir do transcriptoma das células, sendo diretamente influenciado pelo estado fisiológico da célula em determinado momento ${ }^{45}$. A busca por marcadores de viabilidade embrionária tem como foco o secretoma proteômico, definido pelas proteínas produzidas pelo embrião e secretadas para o meio de cultivo ${ }^{46}$. Dessa forma, a caracterização do metaboloma e do proteoma embrionário permitem um maior entendimento dos estágios iniciais da embriogênese, além da possibilidade de refletir a competência embrionária, resultando em aumento das taxas de sucesso de tratamentos de RHA ${ }^{47,48}$. No entanto, apesar dos resultados no campo da pesquisa, a aplicação clínica e a disponibilização de técnicas rotineiramente aplicáveis ainda estão sob investigação e requerem validação ${ }^{49}$.

Diversos aspectos morfológicos avaliados em microscopia de luz têm sido apontados como preditivos do potencial de implantação embrionária, incluindo aqueles relacionados às etapas iniciais do desenvolvimento, como, por exemplo, a morfologia pronuclear e as primeiras clivagens embrionárias. De modo geral, independentemente do dia da transferência, tais critérios parecem apresentar valor preditivo de viabilidade embrionária quando avaliados individualmente ou coletivamente ${ }^{17}$. 
Aspectos morfológicos avaliados no dia da transferência embrionária têm sido priorizados, seja esta realizada no dia 3 ou 5 do desenvolvimento in vitro ${ }^{50,51}$. A seleção embrionária no dia 3 envolve basicamente a avaliação do número de células e do ritmo de clivagem, a avaliação quantitativa e qualitativa da fragmentação citoplasmática, quando presente, a avaliação da simetria entre os blastômeros resultantes das primeiras clivagens e a avaliação da incidência de blastômeros multinucleados e anucleados. Por outro lado, os principais parâmetros avaliados na seleção de embriões em estágio de blastocisto (dia 5 ou 6 do desenvolvimento) incluem grau de expansão da blastocele e padrão de organização da massa celular interna (MCI) e do trofoectoderma (TRF) ${ }^{17}$.

\section{Morfologia pronuclear}

A morfologia pronuclear está diretamente relacionada à qualidade dos gametas, sendo a etapa inicial na qual a normalidade destes pode ser avaliada, fornecendo informações complementares para a seleção embrionária nos estágios iniciais da clivagem. Um número significativo de modelos tem sido proposto para classificação de embriões em estágio de pronúcleo. Principalmente devido a sua clareza e objetividade, os critérios de classificação pronuclear descritos previamente por Tesarik e Greco ${ }^{52}$ e Scott et al. ${ }^{53}$ são os mais utilizados até os dias de hoje. Os parâmetros morfológicos avaliados neste momento estão relacionados às inúmeras transformações observadas durante a nucleogênese em humanos. A avaliação da fertilização costuma ser direta, sendo um oócito considerado fertilizado quando apresenta dois pronúcleos e dois CPs, o que torna questionável a presença de dois CPs, dado que estes podem fragmentar e desintegrar antes do intervalo de avaliação da fertilização ${ }^{17}$.

O valor preditivo da morfologia pronuclear tem sido foco de estudos nos últimos anos. O efeito prognóstico foi abordado $^{52-54}$, bem como a correlação com aneuploidia ${ }^{55}$. Por outro lado, alguns autores não observaram o valor preditivo de tal critério ${ }^{56}$. As alterações mais frequentes observadas em zigotos incluem o tamanho e a posição dos pronúcleos, o número e distribuição dos corpos precursores de nucléolos (CPN) e o halo citoplasmático. Conforme sugestão do consenso, alterações de tamanho ou localização nuclear devem sem registradas, bem como alterações do número de CPN. Pronúcleos apresentando nenhum ou apenas um CPN devem ser associados ao desenvolvimento anormal ${ }^{17}$.

\section{Pronúcleo}

O padrão normal para os pronúcleos feminino e masculino durante a avaliação do zigoto inclui semelhança de tamanho, justaposição e posicionamento central em relação ao citoplasma. $\mathrm{O}$ tamanho dos pronúcleos pode diferir, sendo o masculino o de maior diâmetro. Porém, diferenças de $4 \mu \mathrm{m}$ estão associadas ao comprometimento do desenvolvimento embrionário e a maior incidência de aneuploidias $^{55,57}$. A posição periférica dos pronúcleos ou a não justaposição pode indicar falha nos mecanismos do processo de fertilização, como, por exemplo, a formação do áster e dos microtúbulos ${ }^{58}$. Além disso, o afastamento dos pronúcleos foi previamente associado com pior qualidade embrionária e reduzido potencial de desenvolvimento ${ }^{53}$.

\section{Corpos precursores de nucléolos}

Presentes nos núcleos de todas as células em atividade mitótica, os nucléolos são sítios nos quais os genes ribossomais são transcritos, sendo, portanto, essenciais para síntese proteica. O desenvolvimento dos nucléolos ocorre em áreas denominadas regiões organizadoras de nucléolos (RON), localizadas em pontos específicos dos cromossomos nos quais estão localizados os genes que codificam para os RNA ribossomais (rRNAs). Os nucléolos são caracterizados por um componente fibrilar denso, um centro fibrilar e um componente granular. Oócitos de folículos antrais possuem nucléolos em atividade de síntese de rRNA e proteínas para o crescimento oocitário. Durante a maturação oocitária, a síntese é interrompida e o nucléolo desaparece. Dessa forma, oócitos em Metáfase II e no estágio pronuclear estão caraterizados apenas pela porção do centro fibrilar, sendo denominados $\mathrm{CPN}^{59}$.

A nucleogênese inclui o crescimento, a organização e a fusão dos CPN, eventos dependentes da atividade transcricional pronuclear e da presença de fatores do ooplasma relacionados à maturação oocitária. De modo geral, a fase inicial do desenvolvimento pronuclear é caracterizada por um número elevado de CPN de tamanho relativamente pequeno, distribuídos randomicamente em cada um dos pronúcleos. Por outro lado, a fase mais tardia do desenvolvimento pronuclear é caracterizada por um pequeno número de $\mathrm{CPN}$ de tamanho relativamente maior com distribuição polarizada, sendo observado acúmulo próximo ao local onde um pronúcleo faz contato com o outro. Tais considerações apontam o desenvolvimento dos CPN como um marcador potencial em uma avaliação morfológica não invasiva da qualidade do zigoto. Alterações do padrão dos CPN de embriões em estágio de pronúcleo resultam em efeitos significativos no desenvolvimento embrionário, dado que a falta de polarização, fusão e o restabelecimento de um nucléolo funcional apresenta um efeito negativo na habilidade de crescimento e função celular ${ }^{53,55}$.

\section{Halo citoplasmático}

Após a fertilização é possível observar um padrão diferencial de distribuição do citoplasma no zigoto. A reorganização do citoplasma é dependente de processos da fertilização que resultam em oscilações de cálcio 
caracterizadas por ondas coordenadas temporalmente e espacialmente no citoplasma do oócito. A densa área observada próxima aos pronúcleos está associada com a rotação e o movimento do citoplasma, bem como com a distribuição diferencial de organelas e mitocôndrias, resultando na formação de um halo menos denso na periferia. A distribuição diferencial das mitocôndrias, por sua vez, está relacionada à concentração diferencial de cálcio no citoplasma, afetando a produção de ATP e a necessidade diferencial de energia pelo zigoto ${ }^{60}$.

Muitos estudos confirmaram a importância do halo citoplasmático demonstrando um aumento nas taxas de implantação de embriões para os quais o halo estava presente em estágio de zigoto ${ }^{61}$. A localização das mitocôndrias em uma região diferente da periferia pronuclear pode implicar na distribuição desigual destas para as células-filha de embriões em estágio de clivagem, resultando em depleção da produção de ATP em alguns blastômeros. A ausência de rotação do pronúcleo paterno, diretamente relacionada com a formação do halo citoplasmático, implica no alinhamento anormal da cromatina e/ou no posicionamento inadequado dos centrossomos, resultando em desenvolvimento embrionário anormal. Além disso, a ausência de rotação e o resultado da não polarização do citoplasma podem implicar em distribuição incorreta de produtos gênicos ao longo das sucessivas clivagens ${ }^{62}$. Por outro lado, o efeito desse parâmetro no desenvolvimento embrionário não foi elucidado por outros autores ${ }^{63}$. Além disso, não existem evidências suficientes que suportam o valor preditivo da observação do halo citoplasmático ${ }^{17}$.

\section{Classificação embrionária nos}

dias 2 e 3 do desenvolvimento

Os critérios mais utilizados na seleção de embriões a serem transferidos estão baseados na morfologia embrionária nos dias 2 e 3 do desenvolvimento. De modo geral, a avaliação inclui número de células e ritmo de clivagem, simetria de blastômeros, fragmentação citoplasmática, multinucleação e ausência de núcleo nos blastômeros.

\section{Número de células e ritmo de clivagem}

A ocorrência de divisão celular pode ser considerada o mais importante indicador de viabilidade embrionária. Espera-se observar embriões com 4 células no dia 2 $(44 \mathrm{~h} \pm 1)$ e com 8 células no dia $3(68 \mathrm{~h} \pm 1)$, considerando-se o intervalo de tempo após a ICSI sugerido para avaliação dos embriões ${ }^{17}$.

O comprometimento dos resultados clínicos derivado da transferência de embriões de ritmos de clivagem lento ou rápido foi previamente reportado por diversos autores. De acordo com Alikani et al. ${ }^{64}$, apenas 13,8\% dos embriões com número de células menor do que 7 no dia 3, 41,9\% dos embriões com 7 a 9 células, e $27,5 \%$ dos embriões com número de células maior do que 9 apresentam potencial para formação de blastocistos quando submetidos ao cultivo prolongado. Avaliando retrospectivamente os resultados de transferências realizadas no dia 3 ou 5 do desenvolvimento embrionário, Racowsky et al. ${ }^{65}$ demonstraram que a presença de embriões com 8 células no dia 3 é um forte critério preditivo das taxas de sucesso. Nenhum dos embriões que apresentaram número de células menor do que 8 no dia 3 implantaram quando transferidos no dia 5 do desenvolvimento. Considerando apenas número de células, Racowsky et al. ${ }^{66}$, em outro estudo, demonstraram que embriões de 8 células no dia 3 apresentam maior potencial de implantação (25\%), quando comparados a outros embriões. Em ordem decrescente de viabilidade foram classificados os embriões de mais de 8 células $(18,1 \%)$, seguidos de embriões com 7 células $(10,2 \%)$ e embriões com menos de 7 células $(3,0 \%)$.

O comprometimento do desenvolvimento embrionário de acordo com seu ritmo de clivagem tem sido associado a maior ocorrência de anormalidades cromossômicas em embriões que apresentam alteração em seu ritmo de clivagem ${ }^{67,68}$. Magli et al.$^{67}$ avaliaram o status cromossômico dos estágios de clivagem observados no dia 3 do desenvolvimento embrionário. A incidência de anormalidades cromossômicas foi significativamente menor para o estágio de 7 a 8 células $(50 \%)$, quando comparado a todos os outros estágios. Os índices mais elevados de anomalias cromossômicas foram observados para embriões que apresentaram 2 ou 3 células na manhã do dia $3(94 \%)$ e para aqueles de 4 células que apresentaram divisão de apenas um blastômero em relação ao dia 2 (85\%). Proporções similares de anormalidades cromossômicas foram observadas para embriões de clivagem lenta ( 4 células que apresentaram 2 células no dia 2), embriões moderadamente lentos ( 5 ou 6 células) e embriões de clivagem rápida ( $\geq 9$ células): 74, 73 e 78\%, respectivamente. Além disso, os resultados demonstraram uma taxa de anormalidade de $97 \%$ para embriões que apresentam parada de clivagem do dia 2 para o dia 3 e de $91 \%$ para embriões que apresentam clivagem de apenas 1 blastômero do dia 2 para o dia 3. Este e outros estudos prévios que demonstraram uma associação entre a incidência de aneuploidia e o número de células de embriões em ritmo de clivagem determinaram o status cromossômico por meio da técnica de hibridização in situ por fluorescência (FISH), técnica com falhas descrita na identificação de 20 a 50\% de embriões anormais, quando comparada à técnica de hibridização genômica comparativa $(\mathrm{CGH})^{69}$. Um estudo recente de Kroener et al. ${ }^{68}$ é apontado como o primeiro estudo que teve como objetivo correlacionar parâmetros embrionários e o status cromossômico de embriões determinado pela técnica de CGH. O índice de aneuploidia foi avaliado 
em função do número de células no dia 3, demonstrando maior chance de aneuploidia para embriões com mais de 9 células $(\mathrm{OD}=1,39 ; \mathrm{p}=0,0294)$. A taxa de formação de blastocistos também foi avaliada neste estudo em função do número de células e da constituição cromossômica embrionária. Embriões com ritmo de clivagem acelerado (>9 células) apresentaram maior chance de formação de blastocistos, independentemente da constituição cromossômica. No entanto, dentre os embriões que atingiram o estágio de blastocisto, aqueles com ritmo de clivagem normal ( 6 a 9 células) apresentaram uma menor incidência de aneuploidia ( 54 versus 62\%; $\mathrm{p}=0,096$ ). Dessa forma, o estudo aponta a seleção de blastocistos provenientes de embriões de 6 a 9 células no dia 3 como relacionada a melhores taxas de sucesso, quando comparada à seleção de blastocistos provenientes de embriões com $>9$ células no dia 3. No entanto, apesar da maior sensibilidade e da menor incidência de erros da técnica de CGH em relação ao FISH, o estudo foi baseado na biópsia embrionária no dia 3 do desenvolvimento. A alta incidência de mosaicismo observada em embriões do dia $3^{70}$, bem como a possibilidade de comprometimento do desenvolvimento embrionário decorrente da biópsia (quando realizada nesse dia do desenvolvimento embrionário $)^{71}$, sugere ainda a necessidade de novos estudos a fim de que se estabeleça a associação entre o ritmo de clivagem a e incidência de aneuploidias. Além da maior chance de alteração cromossômica, o ritmo rápido de clivagem foi recentemente correlacionado com perturbações no padrão de imprinting e no metabolismo em modelo de embriões de camundongo ${ }^{72}$.

\section{Simetria de blastômeros}

Devemos destacar que para embriões em estágio de 2, 4 e 8 células, a simetria de blastômeros é representada pela observação de blastômeros de tamanho idêntico. Por outro lado, para embriões em estágio de 3, 5, 6 e 7 células, espera-se observar diferentes tamanhos celulares como indicativo de clivagem normal. Por exemplo, um embrião de 7 células de clivagem normal deve apresentar 1 célula de maior tamanho e 6 menores, sendo que apenas após a divisão da célula de maior tamanho devem ser observados 8 blastômeros de tamanho semelhante. Neste caso, a diferença de tamanho entre os blastômeros é apenas indicativa de assincronia no processo de clivagem das células (a célula maior de um embrião de 7 células encontra-se em ritmo de clivagem mais lento que as demais).

A possibilidade de distribuição irregular de material celular e/ou genético resultando em efeito negativo na viabilidade embrionária tem sido pouco estudada. A maioria dos estudos considera o número de células e a presença de fragmentação como sendo os principais critérios a serem abordados. Porém, alguns autores demonstraram previamente o impacto negativo da assimetria de blastômeros nos resultados obtidos ${ }^{73-75}$. Giorgetti et al. ${ }^{73}$ verificaram uma associação entre assimetria e menores taxas de implantação, porém não diferindo entre irregularidades da forma e do tamanho dos blastômeros. Ziebe et al. ${ }^{74}$ e Hardarson et al. ${ }^{75}$ observaram uma queda significativa do potencial de implantação dos embriões de acordo com alterações do padrão de simetria de blastômeros, considerando-se o tamanho esperado a cada ciclo de divisão celular.

O comprometimento do desenvolvimento embrionário de embriões assimétricos estaria relacionado à ocorrência de alterações na distribuição de material genético, sendo blastômeros originados de embriões assimétricos mais afetados por alterações cromossômicas numéricas $(29,4 \%)$, quando comparados a blastômeros derivados de embriões simétricos $(8,5 \%)^{75}$. Além disso, Alikani et al. ${ }^{76}$ demonstraram que não apenas um maior número de blastômeros desses embriões era aneuploide, mas também que a gravidade da aneuploidia era maior. Além disso, especula-se que a divisão desigual de um blastômero resulte na distribuição inadequada de proteínas, mRNA e organelas para as células-filha. Considerando a ocorrência de polarização de algumas proteínas e produtos gênicos no embrião, o efeito negativo de uma divisão celular desigual seria ainda amplificado.

\section{Fragmentos anucleados}

A fragmentação pode ser definida como uma estrutura extracelular, anuclear, de conteúdo citoplasmático delimitado por membrana ${ }^{76}$. A incidência da fragmentação é difícil de ser avaliada, dado que é necessário inicialmente diferenciar os fragmentos das células e então estimar a proporção relativa do embrião ocupada por esses fragmentos ${ }^{17}$. Johansson et al. ${ }^{77}$ definiram os fragmentos como sendo $<45 \mu \mathrm{m}$ em diâmetro para embriões no dia 2 e $<40 \mu \mathrm{m}$ em diâmetro para embriões no dia 3. Apesar de as causas exatas da fragmentação não estarem estabelecidas, alguns processos celulares parecem estar envolvidos, como apoptose, deficiência de ATP no embrião, perda de blastômero por apoptose devido à alteração cromossômica, ou meramente fragmentos anucleados resultantes da clivagem que serão posteriormente reabsorvidos ${ }^{78}$.

A classificação quantitativa da porcentagem do volume do embrião que é ocupada por fragmentos citoplasmáticos anucleados pode ser associada a uma classificação qualitativa dos fragmentos em cinco grupos (I a V), de acordo com seu tamanho e sua distribuição ${ }^{76}$. Em seu estudo, Alikani et al. $^{76}$ demonstraram pela primeira vez que o grau e o padrão de fragmentação do embrião estavam associados com as taxas de implantação embrionária. Baseados na classificação embrionária do dia 3 de desenvolvimento, os autores relataram que as taxas de implantação para os tipos I e IV de fragmentação diferem de 37,9 para 18,2\%. 
A fragmentação tipo IV apresentou um efeito negativo mesmo quando comprometido um volume restrito do embrião, como demonstrado pelo autor em transferências nas quais apenas embriões apresentando esse tipo de fragmentação foram transferidos. Em outro estudo do mesmo grupo, os autores avaliaram a correlação entre as anormalidades morfológicas de embriões em estágio de clivagem e a habilidade de formação de blastocistos ${ }^{64}$.

Considerando embriões com baixa porcentagem de fragmentação, a avaliação do tamanho e da organização dos fragmentos passa a ser um parâmetro crítico a ser considerado. No entanto, o padrão de fragmentação não pode ser considerado de fácil avaliação, dada a natureza não estática de tais estruturas, além da possibilidade de serem reabsorvidas ou sofrerem lise durante o desenvolvimento embrionário ${ }^{17}$.

Ainda que associação entre fragmentação e a incidência de aneuploidia não tenha sido verificada, sua relação positiva com a ocorrência de mosaicismo foi previamente observada ${ }^{79}$, sugerindo que os fragmentos pudessem conter pedaços cromossômicos resultados de erros no funcionamento dos fusos. Estudos recentes demonstraram que a ocorrência de fragmentação nos estágios iniciais do desenvolvimento embrionário está correlacionada com a erros mitóticos ${ }^{78}$. Tais observações justificariam o impacto desse critério no potencial de desenvolvimento embrionário. Magli et al. ${ }^{67}$ avaliaram a incidência de anormalidades cromossômicas de acordo com a porcentagem e o tipo de fragmentação, considerando o número de células do embrião no dia 3. Os resultados demonstraram que para embriões sem fragmentação ou para aqueles apresentando fragmentação localizada, a incidência de anomalias cromossômicas varia de acordo com o número de células, independentemente do volume do embrião comprometido pela fragmentação. De modo contrário, na presença de fragmentos não localizados, a incidência de anormalidades cromossômicas é significativamente maior para embriões de 7 a 8 células, quando comparados àqueles de mesmo estágio celular que apresentam fragmentação localizada. Para embriões de clivagem lenta ( 4 a 6 células no dia 3), não se observou correlação entre fragmentação e anormalidade cromossômica. Possivelmente, nestes casos, o atraso no ritmo de clivagem é o principal fator relacionado às desordens cromossômicas, conforme previamente discutido. Dessa forma, o estudo sugere que a transferência de embriões de 8 células no dia 3 deve ser priorizada, mesmo na presença de fragmentação e especialmente se esta estiver localizada, e não distribuída, em todo o espaço perivitelínico.

\section{Multinucleação e ausência de núcleos}

Um blastômero contendo mais de um único núcleo em interfase é definido como multinucleado ${ }^{17}$. A avaliação desse parâmetro é mais facilmente realizada no dia 2 do desenvolvimento devido a maior dimensão das células e a melhor acessibilidade destas em razão de seu número reduzido. Segundo o consenso, a ocorrência de multinucleação deve ser registrada para o dia 2 do desenvolvimento, dado o caráter mais informativo desse critério para o dia 2, quando comparado ao dia $3^{17}$. Os relatos da incidência da multinucleação variam consideravelmente. Estudos relatam que a multinucleação incide em cerca de $30 \%$ dos embriões produzidos in vitro, sendo que de 17 a $87 \%$ dos ciclos apresentam pelo menos um embrião multinucleado ${ }^{80}$. As variações na incidência de embriões multinucleados podem estar relacionadas não apenas ao dia de desenvolvimento avaliado, mas também ao momento da observação, visto que apenas blastômeros em interfase apresentam núcleo visível subestimando as taxas de multinucleação. Apesar de a literatura que descreve a multinucleação em embriões ser bastante extensa, a razão da sua ocorrência ainda não foi esclarecida. Os possíveis mecanismos descritos para a ocorrência de multinucleação incluem a cariocinese na ausência da citocinese, a fragmentação parcial do núcleo ou a migração anormal dos cromossomos durante a anáfase da mitose ${ }^{81,82}$.

Especula-se ainda que a multinucleação possa ser induzida pelo protocolo de estimulação ovariana controlada e por condições adversas de cultivo embrionário. A utilização de altas doses de gonadotrofina implica em rápido crescimento folicular na ausência de vascularização adequada, resultando em hipóxia, ou seja, suprimento inadequado de oxigênio para o oócito em processo de maturação ${ }^{83}$. Além disso, esse tipo de protocolo de estímulo está associado ao recrutamento de uma população heterogênea de folículos, aumentando a possibilidade de recuperação de oócitos de competência comprometida ${ }^{84}$. Estudos em humanos demonstraram que os fusos meióticos e mitóticos são estruturas sensíveis a oscilações de temperatura, sendo que os microtúbulos sofrem despolimerização quando em situação de estresse provocada por baixas temperaturas, podendo resultar em embriões cromossomicamente anormais. Considerando-se a fragilidade das estruturas essenciais para a correta divisão celular, sugere-se que as causas da multinucleação possam estar associadas a oscilações de temperatura e $\mathrm{pH}$ durante o cultivo de gametas e embriões ${ }^{81}$.

O potencial negativo da ocorrência de multinucleação no desenvolvimento embrionário pode ser relacionado a erros na replicação e segregação cromossômica, resultando em redução significativa das taxas de sucesso após transferência de embriões multinucleados ${ }^{80}$. Considerando os núcleos de um blastômero multinucleado como entidades separadas, espera-se que a replicação e o condensamento da cromatina ocorram de forma independente, resultando em duas estruturas cromossômicas em um único fuso mitótico e na distribuição cromossômica anormal nas 
células-filha ${ }^{75,85}$. De fato, embriões multinucleados, quando avaliados, apresentaram aumento da incidência de aneuploidia, anormalidades cromossômicas e defeitos na síntese de DNA, conforme relatado inicialmente por Kligman et al. ${ }^{86}$ e posteriormente por Hardarson et al. ${ }^{75}$. A análise da constituição cromossômica de embriões portadores de blastômeros multinucleados demonstrou que $85 \%$ destes apresentavam anormalidades cromossômicas em mais de $50 \%$ de seus blastômeros. Em ambos os estudos, o número de blastômeros cromossomicamente anormais excedia o número de blastômeros comprometidos pela multinucleação, indicando comprometimento genético de todo o embrião. Por outro lado, alguns estudos demonstraram que nem todos os embriões multinucleados apresentam comprometimento de sua constituição cromossômica ${ }^{82}$. A ocorrência de blastômeros binucleados apresenta um menor impacto no comprometimento do desenvolvimento embrionário, visto que uma maior porcentagem desses embriões é caracterizada como euploide, quando comparados com embriões apresentando 3 ou mais núcleos em um dos blastômeros ${ }^{85}$. O estudo de Meriano et al. ${ }^{85}$ considerou, para avaliação do impacto da multinucleação na competência embrionária, dois fenótipos mais comumente encontrados: blastômeros binucleados e multinucleados. Os resultados obtidos demonstraram que embriões mononucleados provenientes de uma coorte comprometida por embriões binucleados não apresentam potencial de desenvolvimento comprometido, diferente do observado para embriões mononucleados provenientes de uma coorte comprometida pela multinucleação. $\mathrm{O}$ estudo sugere que a ocorrência de blastômeros binucleados deve ser considerada uma variável independente restrita ao embrião no qual é detectada. Por outro lado, a multinucleação deve ser indicativo de comprometimento da competência de toda a coorte. Além disso, análise de FISH para cinco cromossomos demonstrou que embriões binucleados apresentam maior incidência de blastômeros normais para os cromossomos testados, quando comparados com embriões multinucleados. Dentre os embriões binucleados avaliados, $32 \%$ foram considerados normais; dentre os multinucleados, apenas 3,7\% tiveram tal classificação.

A significância clínica de blastômeros anucleados ainda não foi elucidada. O estudo de Moriwaki et al. ${ }^{87}$ relatou uma menor chance de implantação para embriões com blastômeros sem núcleo visível no momento da observação. Segundo Palmstierna et al. ${ }^{88}$, considerando o fato de que o desenvolvimento do núcleo de blastômeros envolve fases distintas nas quais este se torna mais ou menos visível e se considerando a observação desse critério em intervalos de tempo preestabelecidos, o fato de o núcleo não ser observado caracteriza um blastômero anucleado. Assim, o impacto desse critério no desenvolvimento embrionário deve estar associado a uma assincronia do ciclo celular entre blastômeros mononucleados e blastômeros anucleados. Dessa forma, um maior comprometimento do embrião por esse critério poderia ainda agravar o efeito observado.

\section{Potencial preditivo de critérios morfológicos}

Faltam estudos na literatura sugerindo de que forma classificar e selecionar embriões apresentando diferentes tipos e graus de variações do padrão ideal de morfologia. Dessa forma, podemos afirmar que não está claro, por exemplo, se um blastocisto que apresentou ritmo de clivagem lenta ou rápida e ausência de fragmentação nos estágios iniciais de clivagem deve ser selecionado para transferência em detrimento de outro que apresentou ritmo de clivagem normal, porém com presença de fragmentação moderada. Ou ainda, se uma variação no tamanho dos blastômeros pode inferir um prognóstico pior do que a ausência de núcleo visível em determinada proporção de blastômeros do embrião. Devemos ressaltar que algumas variáveis podem ser caracterizadas como preditoras independentes do potencial de formação e implantação de blastocistos, porém outras poderão apresentar significância apenas em uma avaliação univariada, perdendo sua significância em modelos multifatoriais. Algumas variáveis sem significância biológica podem demonstrar associação com outras variáveis de real importância, resultando em uma falsa impressão de seu potencial preditivo. Um modelo baseado em análises estatísticas multifatoriais nas quais as variáveis relevantes competirão é apontado como a melhor maneira de se evitar uma classificação subjetiva dos embriões. O modelo incluirá apenas os fatores que apresentarem de forma independente um impacto nas variáveis de sucesso, além de fornecer o correto peso de cada critério. Conforme abordado, poucos estudos optam por uma estratégia de avaliação multidimensional, apesar da necessidade crescente de classificação de embriões baseada em observações diárias pontuais ${ }^{50,89}$.

Dois estudos recentes incluindo mais de $6.000 \mathrm{em}$ briões transferidos tiveram como objetivo a obtenção de um modelo de classificação de embriões de acordo com seu potencial de implantação ${ }^{90,91}$. No entanto, apesar da grande casuística, algumas limitações podem ser apontadas. O estudo de Rhenman et al. ${ }^{91}$ incluiu apenas transferências realizadas no dia 2 do desenvolvimento, sendo que o cultivo prolongado até o estágio de blastocisto teria fornecido maior acurácia na seleção embrionária. Por outro lado, o estudo de van Loendersloot et al..$^{90}$ incluiu apenas parâmetros de morfologia embrionária do dia 3 do desenvolvimento. No entanto, modelos de implantação que incluem variáveis de ambos os dias (2 e 3) têm demonstrado melhor valor preditivo ${ }^{50}$. Além disso, o objetivo do estudo de van Loendersloot et al..$^{90}$ foi 
classificar embriões obtidos no ciclo de tratamento de um casal de acordo com seu potencial de implantação, e não determinar o potencial de implantação de um embrião de modo individual. Dessa forma, as características dos pacientes seriam todas idênticas para esses embriões que eram provenientes de um mesmo casal, excluindo a inclusão de parâmetros como idade no modelo.

Os resultados obtidos nesses estudos devem, de modo geral, ser aplicados com cautela, principalmente pelo fato de serem obtidos e validados a partir de um mesmo banco de dados. O poder estatístico do estudo parece não ser afetado se aplicados testes estatísticos adequados, porém os modelos devem ser validados em populações independentes $^{92}$. A validação externa resulta em um refinamento do modelo a partir de um reajuste do peso de cada variável.

A importância da necessidade de padronização da avaliação morfológica embrionária é atualmente foco da comunidade internacional. O recente encontro de membros da associação internacional de embriologistas ALPHA e do grupo de embriologia da ESHRE teve como objetivo o estabelecimento de critérios e terminologias a serem utilizados na classificação de oócitos, zigotos e embriões que pudessem ser facilmente aplicados na rotina ${ }^{17}$. No entanto, a implementação dos pontos abordados nesse consenso por laboratórios de FIV em todo o mundo ainda é opcional, não sendo abrangida nos relatos de dados anuais da sociedade na qual o laboratório está inserido. Em 2007, dados de morfologia embrionária foram inseridos nos bancos de dados do Sistema de Relato dos Resultados Clínicos da Sociedade Nacional Americana para Tecnologia Reprodutiva Assistida (SART CORS) após o estabelecimento de um sistema de avaliação embrionária. Os dados foram coletados por laboratórios de forma voluntária e avaliados de forma retrospectiva, para validação do sistema com resultados promissores ${ }^{51}$. Porém, dados de morfologia embrionária ainda não fazem parte dos bancos de dados do SART. No Brasil, centros de fertilização assistida acreditados pela REDLARA relatam anualmente dados de todos os procedimentos realizados, porém nenhum dado de morfologia embrionária é incluído ou abordado pelo RLA.

\section{Conclusão}

A falta de consenso na forma de avaliação dos critérios e na determinação da interdependência ou de seu peso relativo em sistemas de avaliação, associada à subjetividade na avaliação e à ampla diversidade de sistemas de classificação embrionária aplicados por diferentes clínicas, às variações inter e intraobservadores e aos diferentes protocolos e meios de cultivo utilizados, implica em resultados contraditórios, tornando extremamente difícil a implementação de um consenso do valor preditivo dos diferentes parâmetros morfológicos avaliados. Além disso, poucos estudos tiveram como foco determinar a interdependência dos parâmetros avaliados ou determinar o peso relativo desses parâmetros em predizer o potencial de desenvolvimento ou implantação de blastocistos. A otimização da seleção embrionária por meio da utilização dos algoritmos nos quais tais variáveis foram incorporadas representa um grande potencial de aumento das taxas de sucesso do tratamento, além de possibilitar a realização da transferência de um número reduzido de embriões, minimizando os riscos derivados de gestações múltiplas. Além disso, o melhor entendimento do potencial preditivo dos critérios de avaliação embrionária poderá auxiliar no estabelecimento de uma estratégia mais uniforme de seleção embrionária entre os diferentes laboratórios.

\section{Agradecimentos}

À Faculdade de Ciências Médicas da Santa Casa de São Paulo e à Coordenação de Aperfeiçoamento de Pessoal de Nível Superior (CAPES).

\section{Referências}

1. Centers of Disease Control and Prevention. 2006-2010 National Survey of Family Growth. Atlanta: CDC; 2010.

2. Centers of Disease Control and Prevention. American Society for Reproductive Medicine. Society for Assisted Reproductive Technology. 2012 assisted reproductive technology fertility clinic success rates report. Atlanta: CDC; 2014.

3. Kupka MS, Ferraretti AP, de Mouzon J, Erb K, D'Hooghe T, Castilla JA, et al. Assisted reproductive technology in Europe, 2010: results generated from European registers by ESHRE†. Hum Reprod. 2014;29(10):2099-113.

4. Zegers-Hochschild F, Schwarze JE, Crosby JA, Musri C, Souza MCB. Assisted reproductive technologies in Latin America: the Latin American Registry, 2012. Reprod Biomed Online. 2015;30(1):43-51.
5. Palermo G, Joris $H$, Devroey $P$, Van Steirteghem AC. Pregnancies after intracytoplasmic injection of single spermatozoon into an oocyte. Lancet. 1992;340(8810):17-8.

6. Semião-Francisco L, Braga DP, Figueira RCS, Madaschi C, Pasqualotto $F F$, laconelli $A$, et al. Assisted reproductive technology outcomes in azoospermic men: 10 years of experience with surgical sperm retrieval. Aging Male. 2010;13(1):44-50.

7. Boulet SL, Mehta A, Kissin DM, Warner L, Kawwass JF, Jamieson DJ. Trends in use of and reproductive outcomes associated with intracytoplasmic sperm injection. JAMA. 2015;313(3):255-63.

8. Conselho Federal de Medicina. Resolução n ${ }^{\circ} 1.957$, de 15 de dezembro de 2010. Dispõe sobre a adoção das normas éticas para a utilização das técnicas de reprodução assistida. Diário Oficial da União, Brasília (2011 jan. 06); Sec. 1:79. 
9. Rienzi L, Balaban B, Ebner T, Mandelbaum J. The oocyte. Hum Reprod. 2012;27 Suppl 1:i2-21.

10. Figueira RC, Braga DP, Semião-Francisco L, Madaschi C, laconelli A Jr, Borges E Jr. Metaphase II human oocyte morphology: contributing factors and effects on fertilization potential and embryo developmental ability in ICSI cycles. Fertil Steril. 2010;94(3): $1115-7$.

11. Gandolfi TA, Gandolfi F. The maternal legacy to the embryo: cytoplasmic components and their effects on early development. Theriogenology. 2001;55(6):1255-76.

12. Ebner T, Moser M, Tews $G$. Is oocyte morphology prognostic of embryo developmental potential after ICSI? Reprod Biomed Online. 2006;12(4):507-12

13. Krisher RL. The effect of oocyte quality on development. J Anim Sci. 2004;82 E-Suppl:E14-23.

14. Swain JE, Pool TB. ART failure: oocyte contributions to unsuccessful fertilization. Hum Reprod Update. 2008;14(5):431-46.

15. Rienzi L, Ubaldi FM, lacobelli M, Minasi MG, Romano S, Ferrero $\mathrm{S}$, et al. Significance of metaphase II human oocyte morphology on ICSI outcome. Fertil Steril. 2008;90(5):1692-700.

16. Van Blerkom J, Henry G. Oocyte dysmorphism and aneuploidy in meiotically mature human oocytes after ovarian stimulation. Hum Reprod. 1992;7(3):379-90.

17. Alpha Scientists in Reproductive Medicine and ESHRE Special Interest Group of Embryology. The Istanbul consensus workshop on embryo assessment: proceedings of an expert meeting. Hum Reprod. $2011 ; 26(6): 1270-83$.

18. Ten J, Mendiola J, Vioque J, de Juan J, Bernabeu R. Donor oocyte dysmorphisms and their influence on fertilization and embryo quality. Reprod Biomed Online. 2007; 14(1):40-8.

19. Ebner T, Moser M, Shebl O, Sommerguber M, Tews G. Prognosis of oocytes showing aggregation of smooth endoplasmic reticulum. Reprod Biomed Online. 2008;16(1):113-8.

20. Rienzi L, Vajta G, Ubaldi F. Predictive value of oocyte morphology in human IVF: a systematic review of the literature. Hum Reprod Update. $2011 ; 17(1): 34-45$.

21. Setti AS, Figueira RCS, Braga DP, Colturato SS, laconelli A Jr, Borges E Jr. Relationship between oocyte abnormal morphology and intracytoplasmic sperm injection outcomes: a meta-analysis. Eur J Obstet Gynecol Reprod Biol. 2011;159(2):364-70.

22. Kahraman S, Yakin K, Dönmez E, Samli H, Bahçe M, Cengiz G, et al. Relationship between granular cytoplasm of oocytes and pregnancy outcome following intracytoplasmic sperm injection. Hum Reprod. 2000;15(1 1):2390-3

23. Ebner T, Yaman C, Moser M, Sommergruber M, Jesacher K, Tews $G$. A prospective study on oocyte survival rate after ICSI: influence of injection technique and morphological features. J Assist Reprod Genet. $2001 ; 18(12): 623-8$.

24. El Shafie $M$, Windt $M L$, Kitshoff $M, M c G$ regor $P$, Sousa $M$, Wranz $P A B$, et al. Ultrastructure of human oocytes: a transmission electron microscopic view. In: El Shafie M, Sousa M, Windt ML, Kruger TF, editors. An atlas of the ultrastructure of human oocytes a guide for assisted reproduction. London: CRC Press; 2000. p. 151-71. (Encyclopedia of Visual Medicine Series).

25. Dozortsev D, Rybouchkin A, De Sutter P, Qian C, Dhont M. Human oocyte activation following intracytoplasmic injection: the role of the sperm cell. Hum Reprod. 1995; 10(2):403-7.

26. Akarsu C, Cağlar G, Vicdan K, Sözen E, Biberoğlu K. Smooth endoplasmic reticulum aggregations in all retrieved oocytes causing recurrent multiple anomalies: case report. Fertil Steril. 2009;92(4): 1496.e1-3.
27. Pelletier C, Keefe DL, Trimarchi JR. Noninvasive polarized light microscopy quantitatively distinguishes the multilaminar structure of the zona pellucida of living human eggs and embryos. Fertil Steril. 2004;81 Suppl 1:850-6.

28. Montag M, Köster M, van der Ven K, van der Ven H. Gamete competence assessment by polarizing optics in assisted reproduction. Hum Reprod Update. $2011 ; 17(5): 654-66$.

29. Mikkelsen AL, Lindenberg S. Morphology of in-vitro matured oocytes: impact on fertility potential and embryo quality. Hum Reprod. 2001;16(8):1714-8.

30. Dandekar P, Talbot P. Perivitelline space of mammalian oocytes: extracellular matrix of unfertilized oocytes and formation of a cortical granule envelope following fertilization. Mol Reprod Dev. 1992;31(2):135-43.

31. Sathananthan AH. Ultrastructure of the human egg. Hum Cell. 1997;10(1):21-38.

32. Farhi J, Nahum $H$, Weissman A, Zahalka N, Glezerman $M$, Levran D. Coarse granulation in the perivitelline space and IVFICSI outcome. J Assist Reprod Genet. 2002;19(12):545-9.

33. Fancsovits $P$, Tóthné ZG, Murber A, Takács FZ, Papp Z, Urbancsek J. Correlation between first polar body morphology and further embryo development. Acta Biol Hung. 2006;57(3):331-8.

34. Ebner T, Shebl O, Moser M, Sommergruber M, Tews G. Developmental fate of ovoid oocytes. Hum Reprod. 2008;23(1):62-6.

35. Ebner T, Moser M, Tews G. Intracytoplasmic sperm injection in zona-free oocytes. J Turk Ger Gynecol Assoc. 2004;5(4):294-8.

36. Van Soom A, Vandaele L, Goossens K, de Kruif A, Peelman L. Gamete origin in relation to early embryo development. Theriogenology. 2007;68 Suppl 1:S131-7.

37. La Sala GB, Nicoli A, Villani MT, Di Girolamo R, Capodanno F, Blickstein I. The effect of selecting oocytes for insemination and transferring all resultant embryos without selection on outcomes of assisted reproduction. Fertil Steril. 2009;91(1):96-100.

38. Herrero J, Meseguer M. Selection of high potential embryos using time-lapse imaging: the era of morphokinetics. Fertil Steril. 2013;99(4):1030-4.

39. Kovacs P. Embryo selection: the role of time-lapse monitoring. Reprod Biol Endocrinol. 2014;12:124.

40. Wong C, Chen AA, Behr B, Shen S. Time-lapse microscopy and image analysis in basic and clinical embryo development research. Reprod Biomed Online. 2013;26(2):120-9.

41. Conaghan J, Chen AA, Willman SP, Ivani K, Chenette PE, Boostanfar $\mathrm{R}$, et al. Improving embryo selection using a computer-automated time-lapse image analysis test plus day 3 morphology: results from a prospective multicenter trial. Fertil Steril. 2013;100(2):412-9.e5.

42. Kirkegaard K, Campbell A, Agerholm I, Bentin-Ley U, Gabrielsen A, Kirk J, et al. Limitations of a time-lapse blastocyst prediction model: a large multicentre outcome analysis. Reprod Biomed Online. 2014;29(2):156-8.

43. Armstrong S, Vail A, Mastenbroek S, Jordan V, Farquhar C. Timelapse in the IVF-lab: how should we assess potential benefit? Hum Reprod. 2015;30(1):3-8.

44. Tachibana C. What's next in 'omics: The metabolome. Science. 2014;345(6203):1519-21.

45. Dominguez DC, Lopes R, Torres ML. Proteomics: clinical applications. Clin Lab Sci. 2007;20(4):245-8.

46. Katz-Jaffe MG, McReynolds S, Gardner DK, Schoolcraft WB. The role of proteomics in defining the human embryonic secretome. Mol Hum Reprod. 2009; 15(5):271-7. 
47. Cortezzi SS, Garcia JS, Ferreira CR, Braga DP, Figueira RCS, laconelli $\mathrm{A} \mathrm{Jr}$, et al. Secretome of the preimplantation human embryo by bottom-up label-free proteomics. Anal Bioanal Chem. $2011 ; 401(4): 1331-9$

48. Cortezzi SS, Cabral EC, Trevisan MG, Ferreira CR, Setti AS, Braga $D P$, et al. Prediction of embryo implantation potential by mass spectrometry fingerprinting of the culture medium. Reproduction. 2013;145(5):453-62.

49. Krisher RL, Schoolcraft WB, Katz-Jaffe MG. Omics as a window to view embryo viability. Fertil Steril. 2015;103(2):333-41.

50. Racowsky C, Ohno-Machado L, Kim J, Biggers JD. Is there an advantage in scoring early embryos on more than one day? Hum Reprod. 2009;24(9):2104-13.

51. Racowsky C, Stern JE, Gibbons WE, Behr B, Pomeroy KO, Biggers JD. National collection of embryo morphology data into Society for Assisted Reproductive Technology Clinic Outcomes Reporting System: associations among day 3 cell number, fragmentation and blastomere asymmetry, and live birth rate. Fertil Steril. 2011 ;95(6):1985-9.

52. Tesarik J, Greco E. The probability of abnormal preimplantation development can be predicted by a single static observation on pronuclear stage morphology. Hum Reprod. 1999; 14(5): 1318-23.

53. Scott L, Alvero R, Leondires M, Miller B. The morphology of human pronuclear embryos is positively related to blastocyst development and implantation. Hum Reprod. 2000;15(1 1):2394-403.

54. Braga DP, Setti AS, Figueira RCS, laconelli A Jr, Borges E Jr. The combination of pronuclear and blastocyst morphology: a strong prognostic tool for implantation potential. J Assist Reprod Genet. 2013;30(10):1327-32.

55. Sadowy S, Tomkin G, Munné S, Ferrara-Congedo T, Cohen J. Impaired development of zygotes with uneven pronuclear size. Zygote. 1998;6(2):137-41.

56. Weitzman VN, Schnee-Riesz J, Benadiva C, Nulsen J, Siano L, Maier D. Predictive value of embryo grading for embryos with known outcomes. Fertil Steril. 2010;93(2):658-62.

57. Gámiz P, Rubio C, de los Santos M, Mercader A, Simón C Remohí J, et al. The effect of pronuclear morphology on early development and chromosomal abnormalities in cleavage-stage embryos. Hum Reprod. 2003;18(1 1):2413-9.

58. Barroso G, Valdespin C, Vega E, Kershenovich R, Avila R, Avendaño $C$, et al. Developmental sperm contributions: fertilization and beyond. Fertil Steril. 2009;92(3):835-48.

59. Fléchon JE, Kopecný V. The nature of the 'nucleolus precursor body' in early preimplantation embryos: a review of fine-structure cytochemical, immunocytochemical and autoradiographic data related to nucleolar function. Zygote. 1998;6(2):183-91

60. Bavister BD, Squirrell JM. Mitochondrial distribution and function in oocytes and early embryos. Hum Reprod. 2000; 15 Suppl 2:189-98.

61. Salumets A, Suikkari AM, Möls T, Söderström-Anttila V, Tuuri T. Influence of oocytes and spermatozoa on early embryonic development. Fertil Steril. 2002;78(5):1082-7.

62. Van Blerkom J, Davis P, Alexander S. Differential mitochondrial distribution in human pronuclear embryos leads to disproportionate inheritance between blastomeres: relationship to microtubular organization, ATP content and competence. Hum Reprod. 2000;15(12):2621-33.

63. Demirel LC, Evirgen O, Aydos K, Unlü C. The impact of the source of spermatozoa used for ICSI on pronuclear morphology. Hum Reprod. 2001;16(1 1):2327-32.

64. Alikani M, Calderon G, Tomkin G, Garrisi J, Kokot M, Cohen J. Cleavage anomalies in early human embryos and survival after prolonged culture in-vitro. Hum Reprod. 2000;15(12):2634-43.
65. Racowsky C, Jackson KV, Cekleniak NA, Fox JH, Hornstein MD, Ginsburg ES. The number of eight-cell embryos is a key determinant for selecting day 3 or day 5 transfer. Fertil Steril. 2000;73(3):558-64.

66. Racowsky C, Combelles CM, Nureddin A, Pan Y, Finn A, Miles $L$, et al. Day 3 and day 5 morphological predictors of embryo viability. Reprod Biomed Online. 2003;6(3):323-31.

67. Magli MC, Gianaroli L, Ferraretti AP, Lappi M, Ruberti A, Farfalli V. Embryo morphology and development are dependent on the chromosomal complement. Fertil Steril. 2007;87(3):534-41.

68. Kroener LL, Ambartsumyan G, Pisarska MD, Briton-Jones C, Surrey $M$, Hill D. Increased blastomere number in cleavagestage embryos is associated with higher aneuploidy. Fertil Steril. 2015; 103(3):694-8.

69. Fragouli E, Katz-Jaffe M, Alfarawati S, Stevens J, Colls P, Goodall $\mathrm{NN}$, et al. Comprehensive chromosome screening of polar bodies and blastocysts from couples experiencing repeated implantation failure. Fertil Steril. 2010;94(3):875-87.

70. Taylor TH, Gitlin SA, Patrick JL, Crain JL, Wilson JM, Griffin DK. The origin, mechanisms, incidence and clinical consequences of chromosomal mosaicism in humans. Hum Reprod Update. 2014;20(4):571-81.

71. Scott RT Jr, Upham KM, Forman EJ, Zhao T, Treff NR. Cleavagestage biopsy significantly impairs human embryonic implantation potential while blastocyst biopsy does not: a randomized and paired clinical trial. Fertil Steril. 2013;100(3):624-30.

72. Market Velker BA, Denomme MM, Mann MR. Loss of genomic imprinting in mouse embryos with fast rates of preimplantation development in culture. Biol Reprod. 2012;86(5):143, 1-16.

73. Giorgetti C, Terriou P, Auquier P, Hans E, Spach JL, Salzmann $J$, et al. Embryo score to predict implantation after in-vitro fertilization: based on 957 single embryo transfers. Hum Reprod. $1995 ; 10(9): 2427-31$.

74. Ziebe S, Petersen K, Lindenberg S, Andersen AG, Gabrielsen A, Andersen AN. Embryo morphology or cleavage stage: how to select the best embryos for transfer after in-vitro fertilization. Hum Reprod. 1997; 12(7):1545-9.

75. Hardarson T, Hanson C, Siögren A, Lundin K. Human embryos with unevenly sized blastomeres have lower pregnancy and implantation rates: indications for aneuploidy and multinucleation. Hum Reprod. 2001;16(2):313-8.

76. Alikani M, Cohen J, Tomkin G, Garrisi GJ, Mack C, Scott RT. Human embryo fragmentation in vitro and its implications for pregnancy and implantation. Fertil Steril. 1999;71(5):836-42.

77. Johansson $M$, Hardarson $T$, Lundin K. There is a cutoff limit in diameter between a blastomere and a small anucleate fragment. J Assist Reprod Genet. 2003;20(8):309-13.

78. Stensen $M H$, Tanbo TG, Storeng R, Ảbyholm T, Fedorcsak P. Fragmentation of human cleavage-stage embryos is related to the progression through meiotic and mitotic cell cycles. Fertil Steril. 2015;103(2):374-81.e4.

79. Munné $S$, Cohen J. Chromosome abnormalities in human embryos. Hum Reprod Update. 1998;4(6):842-55.

80. Van Royen E, Mangelschots K, Vercruyssen M, De Neubourg D, Valkenburg $M$, Ryckaert $G$, et al. Multinucleation in cleavage stage embryos. Hum Reprod. 2003;18(5):1062-9.

81. Pickering SJ, Taylor A, Johnson MH, Braude PR. An analysis of multinucleated blastomere formation in human embryos. Hum Reprod. 1995;10(7): 1912-22.

82. Staessen C, Van Steirteghem A. The genetic constitution of multinuclear blastomeres and their derivative daughter blastomeres. Hum Reprod. 1998;13(6):1625-31. 
83. Van Blerkom J, Antczak M, Schrader R. The developmental potential of the human oocyte is related to the dissolved oxygen content of follicular fluid: association with vascular endothelial growth factor levels and perifollicular blood flow characteristics. Hum Reprod. 1997; 12(5): 1047-55.

84. Figueira RCS, Setti AS, Braga DPAF, laconelli A Jr, Borges E Jr. Blastomere multinucleation: contributing factors and effects on embryo development and clinical outcome. Hum Fertil (Camb). 2010;13(3): 143-50.

85. Meriano J, Clark C, Cadesky K, Laskin CA. Binucleated and micronucleated blastomeres in embryos derived from human assisted reproduction cycles. Reprod Biomed Online. 2004;9(5):51 1-20.

86. Kligman I, Benadiva C, Alikani M, Munne S. The presence of multinucleated blastomeres in human embryos is correlated with chromosomal abnormalities. Hum Reprod. 1996;1 1 (7):1492-8.

87. Moriwaki T, Suganuma N, Hayakawa M, Hibi H, Katsumata $Y$, Oguchi $\mathrm{H}$, et al. Embryo evaluation by analysing blastomere nuclei. Hum Reprod. 2004;19(1):152-6.

88. Palmstierna M, Murkes D, Csemiczky G, Andersson O, Wramsby $\mathrm{H}$. Zona pellucida thickness variation and occurrence of visible mononucleated blastomers in preembryos are associated with a high pregnancy rate in IVF treatment. J Assist Reprod Genet. 1998; 15(2):70-5.

89. Sjöblom P, Menezes J, Cummins L, Mathiyalagan B, Costello MF. Prediction of embryo developmental potential and pregnancy based on early stage morphological characteristics. Fertil Steril. 2006;86(4):848-61.

90. van Loendersloot $L$, van Wely M, van der Veen F, Bossuyt P, Repping S. Selection of embryos for transfer in IVF: ranking embryos based on their implantation potential using morphological scoring. Reprod Biomed Online. 2014;29(2):222-30.

91. Rhenman A, Berglund L, Brodin T, Olovsson M, Milton K, Hadziosmanovic $N$, et al. Which set of embryo variables is most predictive for live birth? A prospective study in 6252 single embryo transfers to construct an embryo score for the ranking and selection of embryos. Hum Reprod. 2015;30(1):28-36.

92. te Velde ER, Nieboer D, Lintsen AM, Braat DD, Eijkemans M, Habbema JD, et al. Comparison of two models predicting IVF success; the effect of time trends on model performance. Hum Reprod. $2014 ; 29(1): 57-64$ 